\title{
HIRAYAMA DISEASE
}

Shaik Sulaiman Meeron'1, Senthilkumaran Arjunan², Murugarajan Singaram ${ }^{3}$

${ }^{1}$ Associate Professor, Department of Medicine, Chengalpattu Medical College. ${ }^{2}$ Assistant Professor, Department of Medicine, Chengalpattu Medical College. 3 Junior Resident, Department of Medicine, Chengalpattu Medical College.

\section{ABSTRACT}

Hirayama's disease, also known as Monomelic Amyotrophy (MMA), juvenile non-progressive amyotrophy, Sobue disease. It is rare and benign condition. It is a focal, lower motor neuron type of disorder, which occurs mainly in young males. Age of onset, it is first seen most commonly in people in their second and third decades. Geographically, it is seen most commonly in Asian countries like India and Japan. Cause of this disease is unknown in most cases. MRI of cervical spine in flexion is the investigation of choice, which will reveal the cardinal features of Hirayama disease.

\section{CASE REPORT}

20 years old male came with the complaints of tremors of both hands more of right hand and weakness and wasting of right hand, which is slowly progressive for past 6 months. Lower limbs had no abnormality with normal deep tendon reflexes. On examination, there was wasting and weakness of hypothenar and interosseous muscles of right hand. MRI showed thinning of cord from C5 to C7 level. Proximal epidural fat and tiny flow voids with anterior migration of the posterior dural layer at C5-7 level on flexion MRI. Based on these features a diagnosis of focal amyotrophy was made. A cervical collar was prescribed and patient is under regular follow-up.

\section{CONCLUSION}

Hirayama disease is a rare self-limiting disease. Early diagnosis is necessary as the use of a simple cervical collar which will prevent neck flexion, has been shown to stop the progression.

\section{KEYWORDS}

Hirayama's Disease, Monomelic Amyotrophy, Juvenile Non-Progressive Amyotrophy, Sobue Disease.

HOW TO CITE THIS ARTICLE: Meeron SS, Arjunan S, Singaram M. Hirayama disease. J. Evolution Med. Dent. Sci. 2016;5(38): 2353-2355, DOI: 10.14260/jemds/2016/546

\section{INTRODUCTION}

20 years old male came with the complaints of tremulousness of both hands, more of right hand for past 6 months. Patient is having difficulty in using right hand for past 6 months in the form of difficulty in mixing food. Patient had also noticed wasting of right hand. The wasting and weakness is slowly progressive for past 6 months. No history suggestive of involvement of proximal muscles of upper limbs and both the lower limbs. No history of muscle cramps, pain or loss of sensation. No history suggestive of involvement of cranial nerves. No history of bladder and bowel involvement. No history of fever, convulsions, headache and vomiting. No history of trauma, fever, poliomyelitis, exposure to heavy metals or toxins in the past. There was no family history of similar complaints.

On examination, patient had stable vitals. Patient conscious, higher mental functions are normal. His cranial nerves and sensory system examination were normal. Motor examination showed wasting of hypothenar and interosseous muscles of right hand. Hand grip reduced on right side.

Financial or Other, Competing Interest: None.

Submission 28-02-2016, Peer Review 22-04-2016,

Acceptance 28-04-2016, Published 12-05-2016.

Corresponding Author:

Dr. Shaik Sulaiman Meeron,

Associate Professor,

Department of Medicine,

Chengalpattu Medical College,

Chengalpattu.

E-mail: sulaiman14@rediffmail.com

DOI: $10.14260 /$ jemds $/ 2016 / 546$
Power of dorsal and palmar interosseous, flexor digiti minimi, opponens digiti minimi were reduced. Deep tendon reflexes were not exaggerated with bilateral plantar flexor. Patient is having fine tremors of both hands. No other involuntary movements. Sensory system, coordination, gait were all normal.

\section{Blood Investigations}

Complete haemogram, renal function test, liver function test, thyroid profile, HBsAg, anti HCV, serum CPK were all normal. Plain cervical spine X-ray normal except for loss of cervical lordosis.

\section{Nerve Conduction Study}

CMAP (Continued Muscle Action Potential) reduced in right ulnar nerve. F-waves not obtained. Other nerves normal.

\section{Electromyography-Normal Study}

MRI spine-loss of cervical lordosis. Focal thinning of the spinal cord seen at C5-7 level without significant altered signal intensity. Proximal epidural fat and tiny flow voids with anterior migration of the posterior dural layer at C5-7 level on flexion MRI. 


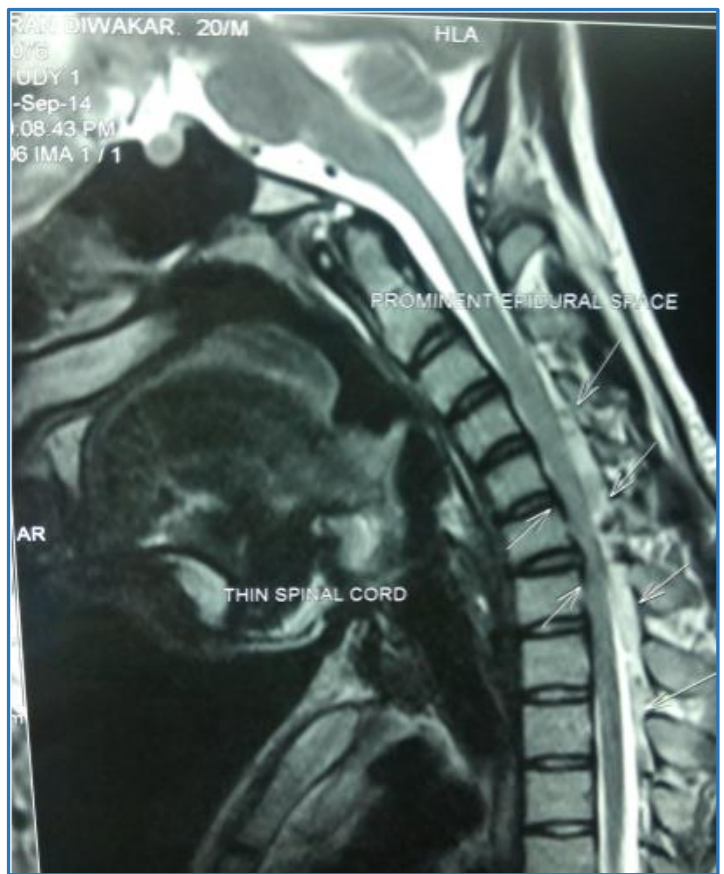

Fig. 1: MRI Neck in Flexion shows Forward Displacement of Posterior Wall and Crescent Shaped Mass in Posterior Epidural Space

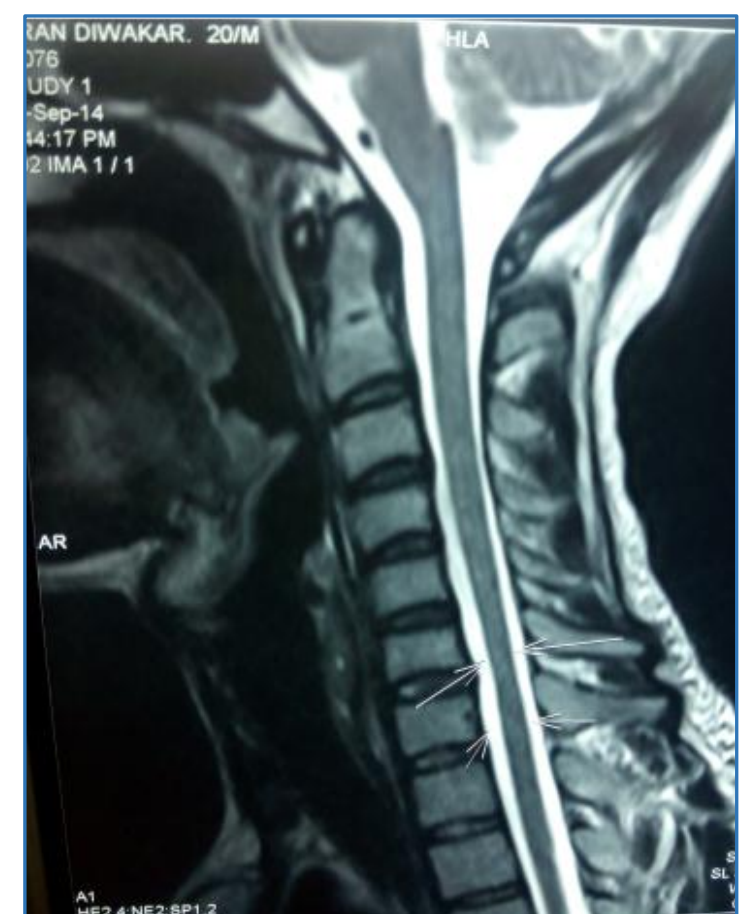

Fig. 2: MRI Neck showing Atrophy of Lower Cervical Cord in Neutral Position

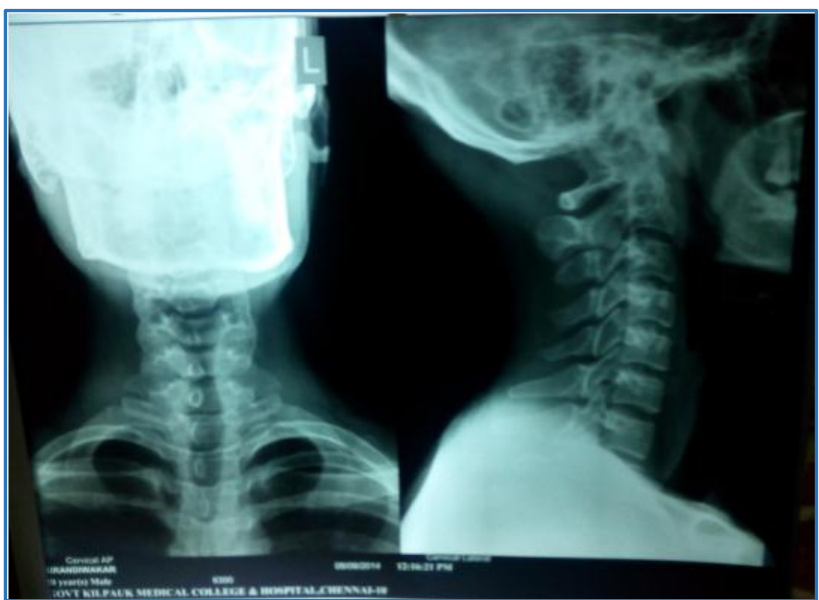

Fig. 3: X-Ray Neck showing Mild Scoliosis

\section{DISCUSSION}

Hirayama disease is characterised by focal amyotrophy with unilateral or asymmetric bilateral weakness and wasting of muscles innervated by C7, C8 and T1. It is an insidious onset, chronic, often self-limiting disorder with male preponderance seen between the ages of 15 and 25 years.

Hirayama et al first reported this disease in the year 1959, but pathologic study was not done till 1982, because of its benign course. At pathologic examination, these authors found the lesions only in the anterior horns of the spinal cord from C5 to T1, particularly marked at C7 and C8.[1] Most commonly seen in Japan and India.[2,3]

The pathogenesis of the disorder is unknown-probable causes suggest that an imbalanced growth between the patient's vertebral column and spinal canal contents. ${ }^{[4]}$ This imbalanced growth will cause disproportional length between the patient's vertebral column and the spinal canal contents, which will cause a "tight dural sac" or "overstretch of the cord" in the neutral position. In neck extension, the dura mater of the cervical spine is slack and thrown into transverse fold. In neck flexion, the dura becomes tighter because the length of the cervical canal increases as the neck moves from extension to flexion. The difference in length between extension and flexion from $\mathrm{T} 1$ to the top of the atlas is $1.5 \mathrm{~cm}$ at the anterior wall and $5 \mathrm{~cm}$ at the posterior wall. Normally, the slack of the dura can compensate for the increased length in flexion; therefore, the dura can still be in close contact with the walls of the spinal canal without anterior displacement.

In Hirayama disease the dural canal is no longer slack in extension, because of an imbalance in growth of the vertebrae and the dura mater. Therefore, a tight dural canal is formed, which cannot compensate for the increased length of the posterior wall during flexion.[5,6] This causes an anterior shifting of the posterior dural wall with consequent compression of the cord. This compression may cause microcirculatory disturbances in the territory of the anterior spinal artery or in the anterior portion of the spinal cord. The chronic circulatory disturbance resulting from repeated or sustained flexion of the neck may produce necrosis of the anterior horns, which are most vulnerable to ischemia.

In patients with Hirayama disease, conventional X-ray of the cervical spine usually show no specific abnormalities except straight alignment or scoliosis. Myelography may show the forward movement of the posterior dural wall when 
the neck is flexed; however, myelography is difficult to perform, as it is difficult to retain the contrast medium in the cervical subarachnoid space when the neck is flexed, regardless of the patient's position. MRI studies in neck flexion, which are easy to obtain will show the forward displacement of the posterior wall and a well-enhanced crescent-shaped mass in the posterior epidural space of the lower cervical canal. This mass is thought to represent congestion of the posterior internal vertebral venous plexus rather than vascular malformations or tumours, because it vanishes once the neck returns to a neutral position. MRI shows atrophy of the lower cervical cord in a neutral position and there will be abnormal cervical curvature (straight or kyphotic) and loss of attachment between the posterior dural sac and subjacent lamina, which is most valuable in Hirayama disease.

\section{CONCLUSION}

Even though Hirayama disease is a rare self-limiting disease, early diagnosis is necessary. Use of a simple cervical collar to prevent neck flexion has been shown to halt the progression of the disease. Diagnosis of Hirayama disease is mainly based on flexion MRI of cervical spine. Asymmetry is one of the most characteristic findings of this disease, both clinically and radiologically. Thus, in cases of adolescent onset slowly progressive distal upper limb weakness followed by stabilization with neurogenic changes in the EMG and the findings of asymmetric cord atrophy on regular non-flexion cervical spine MRI studies, especially at the lower cervical cord, one should keep in mind the diagnosis of Hirayama disease. When this sign is seen, a flexion MRI study should be performed to confirm the diagnosis.

\section{REFERENCES}

1. Hirayama $\mathrm{K}$, Tomonaga $\mathrm{M}$, Kitano $\mathrm{K}$, et al. Focal cervical poliopathy causing juvenile muscular atrophy of distal upper extremity: a pathological study. J Neurol Neurosurg Psychiatry 1987;50(3):285-90.

2. Gourie-Devi M. Monomelic amyotrophy of upper or lower limbs. In: Wisen AA, Shaw PJ, editors. Handbook of clinical neurology. Elsevier, BV. 2007;207-27.

3. Pal Pramod K, Atchayaram Nalini, Goel Gaurav. Central motor conduction in brachial monomelic amyotrophy. Neurology India 2008;56(4):438-43.

4. Hirayama K, Tokumaru Y. Cervical dural sac and spinal cord in juvenile muscular atrophy of distal upper extremity. Neurology 2000;54(10):1922-6.

5. Hirayama K. Non-progressive juvenile spinal muscular atrophy of the distal upper limb (Hirayama's disease). In: De Jong JMBV, ed. Handbook of clinical neurology. Amsterdam, the Netherlands: Elsevier. 1991;15:107-20.

6. Hirayama K. Juvenile non-progressive muscular atrophy localized in hand and forearm: observation in 38 cases. Rinsho Shinkeigaku 1972;12(7):313-24. 